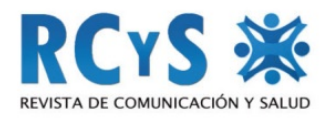

Enviado 28 de mayo de 2017 Aprobado 8 de julio 2017

\title{
PERCEPCIÓN DE RIESGO ANTE EVENTOS DE SALUD URBANA EN TRABAJADORES DE EPIDEMIOLOGÍA EN PARAGUAY
}

\author{
Perception of risk before urban health events in epidemiology workers in \\ Paraguay \\ Patricia Lima Pereira ${ }^{1}$, Edgar Tullo \\ Dirección General de Vigilancia de la Salud, Ministerio de Salud Pública y \\ Bienestar Social. Asunción, Paraguay
}

\begin{abstract}
Resumen
La comunicación de riesgo necesita evaluar la reacción emocional de la audiencia frente a la amenaza específica. En este estudio se analizó la relación entre la percepción de riesgo (subjetiva) ante eventos de salud urbana y un índice de evaluación de riesgo (objetivo) que se construyó a partir de la probabilidad y gravedad de los mismos eventos, en funcionarios de la red de vigilancia de la salud de Paraguay $(n=32)$. Un $87,5 \%$ eran universitarios y $30 \%$, epidemiólogos. Entre las primeras opciones de información se citaron las redes sociales $(78,1 \%)$ y la prensa online $(75 \%)$. En cinco de los siete eventos evaluados (dengue, chikungunya, obesidad, VIH e infecciones respiratorias agudas), la percepción de riesgo fue significativamente más baja que la evaluación del riesgo a nivel colectivo (Kruskal-Wallis, $p<0,05$ ) y en uno de los eventos (asaltos), la percepción fue más baja que la evaluación de riesgo personal. Se identificó que "vulnerabilidad", "temor" y la "posibilidad de catástrofe" estaban asociados a mayor percepción de riesgo (Mann-Whitney, $p<0,05$ ).
\end{abstract}

Palabras clave: Comunicación de riesgo; Medición de Riesgo; Percepción de riesgo; Salud Urbana

\begin{abstract}
Risk communication should assess the emotional reaction of the audience against specific threats. The relationship between risk perception to events of urban health and a risk assessment index that is constructed from the likelihood and severity of these events was analyzed in health surveillance officials in Paraguay $(n=32) .87 .5 \%$ were graduates and $30 \%$, epidemiologists. Social networks $(78.1 \%)$ and online media $(75 \%)$ were cited as the first choices of information. In five of the seven evaluated events (dengue, chikungunya, obesity, HIV and acute respiratory infections), risk perception was significantly lower than the collectively risk assessment (Kruskal-Wallis, $p<0.05$ )
\end{abstract}

\footnotetext{
${ }^{1}$ Autor de contacto: Patricia Lima patricialimap@gmail.com
} 
Percepción de riesgo ante eventos de salud urbana en trabajadores de epidemiología en Paraguay

and in one of the events (attacks), the perception was lower than the personal risk assessment. "Vulnerability", "fear" and "possible catastrophe" were associated with greater perceived risk (Mann-Whitney test, $p<0.05$ ).

Key words: Risk communication, risk assessment, risk perception, urban health

\section{Como citar el artículo}

Lima Pereira, P., Tullo, E. (2017). Percepción de riesgo ante eventos de salud urbana en trabajadores de epidemiología en Paraguay. Revista de Comunicación y Salud, vol. 7 , 61-79. Recuperado

http://revistadecomunicacionysalud.org/index.php/rcys/article/view/122

Los autores agradecen especialmente a Antonieta Rojas de Arias, Margarita Villafañe, Cristina Maña, Águeda Cabello y Diana Rodríguez por sus contribuciones para este trabajo.

\section{INTRODUCCIÓN}

Desde finales del siglo $\mathrm{XX}$ el mundo ha registrado un acelerado proceso de urbanización y se convirtió en mayoritariamente urbano desde el 2007 (Harpham, 2009). En el 2014, el 54\% de la población mundial residía en áreas urbanas y se prevé que para 2050 llegará al 66\% (ONU, 2014), lo que representa un desafío para las políticas públicas de salud y desarrollo sostenible. La salud urbana es un tema complejo que no está solo determinado por factores materiales, y de acceso a servicios básicos e infraestructura (Lee, 2011). Los principales obstáculos para mejorar la salud urbana no son técnicos o financieros, sino que están relacionados con la gobernanza y la organización de la sociedad civil, donde la comunicación es fundamental (Dye, 2008; Fumkin et al, 2004).

Vivir en la ciudad no es garantía de acceso a servicios de salud. Dentro de las ciudades es donde se observan las mayores inequidades (Berzins et al, 2014; Montgomery, 2009). Las ciudades aumentan el riesgo de ciertas enfermedades infecciosas. Un ejemplo clásico del riesgo existente en las ciudades fue la epidemia de cólera en Paris, en 1830 (Kudlick, 1988). Asimismo, en 2010, la Organización Mundial de la Salud destacó el aumento de la incidencia de la tuberculosis en las zonas urbanas (WHO, 2010). Sumado a esto, la destrucción de bosques y hábitat tradicionales de insectos y animales, obliga a estas especies a desplazarse a nuevas zonas. Es así que los seres humanos conviven cada vez más con vectores de enfermedades que se han adaptado exitosamente para vivir en el hábitat urbano (Reis et al, 2008). El hacinamiento y el desplazamiento de la población vinculados al estilo de vida urbano, aumenta el riesgo de estar en contacto con nuevos virus y parásitos. El dengue, el chikungunya y otras enfermedades vectoriales constituyen problemas de salud pública importantes para numerosas zonas urbanas en el mundo.

En este contexto de riesgo, es donde la comunicación cumple una especial importancia, ya que puede permitir el aprovechamiento práctico del conocimiento científico, y la apropiación de la ciencia por parte de la sociedad, con el objetivo de 
Percepción de riesgo ante eventos de salud urbana en trabajadores de epidemiología en Paraguay

prevenir enfermedades, mantener y recuperar la salud. La comunicación de riesgo es más eficaz cuando se integra con el análisis y la gestión de riesgos, contrastado la amenaza con un componente subjetivo expresado en la reacción emocional de la audiencia (OMS, 2015; Brennan y Gutierrez, 2011). Para mayor claridad en el análisis, es importante entender la diferencia entre riesgo y percepción de riesgo. Riesgo en epidemiología es la probabilidad de un evento (Gordis, 2014). En el manejo integral del riesgo, se considera al riesgo como la relación entre la gravedad de la amenaza y la vulnerabilidad de la población expuesta (IPCC, 2013). A su vez, la vulnerabilidad es definida como la predisposición a verse afectados de manera adversa por un evento (IPCC, 2013). Se trata por tanto de una medida objetiva, posible de cuantificar. En tanto, la percepción de riesgo, es una construcción social que está determinada por el juicio subjetivo que la gente hace sobre las características y la gravedad de una amenaza (Sandman, 2003).

\subsection{Medición del riesgo}

Desde finales del siglo $\mathrm{XX}$, los investigadores han estudiado el riesgo de manera intensiva y desde diferentes perspectivas. Algunos autores plantean que en la cultura contemporánea el riesgo es ubicuo, filtrado a través de una serie de actividades, prácticas y experiencias (Mythen and Walklate, 2006; Adam 2000). Existen diversos modelos para medir el riesgo, más o menos especializado (Flaus, 2012; Renn, 1992; Ostrom et al, 2012) y la vulnerabilidad frente al riesgo (Laugesen et al, 2010). Algunos métodos simplificados pueden llegar a aproximaciones útiles, incluso con pocos datos disponibles, utilizando principalmente sólo tablas o diagramas (Proske, 2008). Entre estas técnicas de evaluación rápida se cita la Tabla de Evaluación de Riesgos de la NASA, la Serie Australiana, la de GTZ, entre otras. Todas usan una puntuación de estimación aproximada de un riesgo, considerando la probabilidad y la gravedad de un evento, y son útiles por la facilidad de su aplicación (Proske, 2008).

\subsection{Percepción de riesgo}

La percepción de riesgo es un componente subjetivo fundamental para diseñar estrategias de comunicación (Slovic, 2000), y se relaciona con valores, procesos, poder y confianza. La discrepancia entre la evaluación subjetiva y objetiva pueden explicarse con diferentes aproximaciones que no sólo las preferencias individuales, sino también las condiciones sociales y culturales (Proske, 2008). La ponderación de los elementos psicológicos, personales, culturales y sociales en el juicio subjetivo es aún objeto de debate.

La percepción del riesgo depende mucho de cómo se comunica la información sobre el origen del riesgo, los mecanismos psicológicos para el procesamiento de la incertidumbre y las experiencias previas de peligro. Las personas construyen su propia realidad y evalúan los riesgos de acuerdo a sus percepciones subjetivas (Ostrom et al, 2012). Dentro del paradigma psicométrico, estudios demuestran que el riesgo percibido se ve afectado por la falta de control, temor, potencial catastrófico, consecuencias fatales, y la distribución no equitativa de los riesgos y beneficios; entre otros. Covello y 
Percepción de riesgo ante eventos de salud urbana en trabajadores de epidemiología en Paraguay

Merkhoher (1993) citan diversos factores que influyen en aumentar la percepción de riesgo. Entre ellos se incluye:

- Voluntariedad: Riesgos involuntarios son percibidos como mayores riesgos en comparación con los riesgos voluntarios.

- Control: Riesgos bajo el control de los demás son percibidos como mayores riesgos en comparación con los riesgos bajo control del individuo.

- Familiaridad: Riesgos desconocidos son percibidos como mayores riesgos en comparación con los riesgos conocidos.

- Equidad: Riesgos distribuidos de forma desigual son percibidos como mayores riesgos en comparación con los riesgos distribuidos uniformemente.

- Beneficios: Riesgos con un beneficio poco claro son percibidos como mayores riesgos en comparación con los riesgos con un claro beneficio.

- Entendimiento: Riesgos difícil de entender son percibidos como mayores riesgos en comparación con los riesgos claramente comprensibles.

- Incertidumbre: Riesgos desconocidos son percibidos como mayores riesgos en comparación con los riesgos conocidos.

- Temor: Riesgos que generan fuertes sentimientos como el miedo son percibidos como mayores riesgos en comparación con los riesgos que no crean sentimientos tan fuertes.

- Desconfianza: Los riesgos fiduciarios conectados a las personas o instituciones con baja credibilidad son percibidos como mayores riesgos en comparación con los riesgos vinculados a las personas u organizaciones confiables.

- Reversibilidad: Riesgos con efectos irreversibles son percibidos como mayores riesgos en comparación con los riesgos sin tales efectos.

- Interés personal: Riesgos a nivel personal son percibidos como mayores riesgos en comparación con los riesgos más impersonales.

- Ética y moral: Riesgos relacionadas con las condiciones éticas o morales bajas son percibidos como mayores riesgos en comparación con los riesgos vinculados a las altas condiciones éticas o morales.

- Víctimas conocidas: Riesgos con múltiples con víctimas identificables son percibidos como mayores riesgos en comparación con los riesgos con víctimas estadísticos.

- Catástrofes: Los riesgos con potencial de crear víctimas con concentraciones espaciales o temporales son percibidos como mayores en comparación con los riesgos que son difusa a través del tiempo y el espacio.

\section{OBJETIVO DE INVESTIGACIÓN}

El objetivo del presente estudio es comparar la percepción y evaluación de riesgo con respecto a eventos relevantes para la salud urbana en personal que tiene vínculo con las acciones de vigilancia epidemiológica en Paraguay.

\section{MÉTODO}

Se propone un estudio transversal, de tipo descriptivo y relacional. 
Percepción de riesgo ante eventos de salud urbana en trabajadores de epidemiología en Paraguay

\subsection{Muestreo}

El universo del presente estudio contempló a todos los funcionarios de los niveles regionales y nacionales de la red de vigilancia de la Salud de Paraguay, compuesta por un total aproximado de 300 funcionarios. Para el estudio se remitió por correo electrónico la invitación para participar de la encuesta online a 164 funcionarios, de los que se tuvo acceso al correo electrónico. En la invitación, se explicaron los objetivos de la encuesta, se informó que se trataba de una encuesta voluntaria y que las respuestas serían confidenciales. Se envió un recordatorio a la semana del primer envío. Al tratarse de un acto voluntario, la participación de la encuesta, fue tomada como un consentimiento informado.

\subsection{Desarrollo de instrumentos de recogida de datos}

Se construyó un cuestionario para la recolección de información sobre percepción y evaluación de riesgo, utilizando el formato de formulario digital de Google, que permite su envío y carga por internet directamente por parte del participante que recibe el enlace, y la fácil disponibilidad de los datos cargados. Tiene la ventaja adicional de que el cuestionario se puede llenar y enviar también desde el teléfono del participante.

Previamente se realizó una revisión bibliográfica sobre los temas de salud pública en entornos urbanos, medición y gestión de riesgo, comunicación de riesgo y percepción de riesgo, con el objetivo de identificar instrumentos para medir la percepción de riesgo de la población. Dado que no se identificó ninguna escala o herramienta disponible para medir la percepción de riesgo en relación a la salud urbana, se propuso, por tanto, adaptar una a los efectos de este estudio.

Se recurrió a adecuar una matriz de evaluación de riesgos aproximados (NASA Risk Assessment Table), donde se relacionan las variables de severidad y probabilidad de un evento, con el objetivo de una evaluación rápida para la implementación de medidas de contención (Proske, 2008).

Considerando que la evaluación de riesgo se debe realizar sobre situaciones concretas y específicas, se seleccionaron siete eventos relacionados con el estilo de vida urbano: Ébola, enfermedad por el virus del dengue, enfermedad por el virus del Chikungunya, Obesidad, Infección por VIH, Infecciones Respiratorias Agudas Graves (IRAG), y Asaltos violentos. Se eligieron las mismas, porque se tratan de problemas de salud con alguna probabilidad de incidencia en población urbana y con cierta severidad para ser considerados globalmente como problemas de salud pública y su vínculo con el desarrollo sostenible. Cada uno de los eventos presenta cierta peculiaridad que podría determinar una mayor o menor percepción de riesgo en la población, según se indica en la tabla 1.

La herramienta utilizada como base (Proske, 2008) propone una evaluación aproximada del riesgo del evento de acuerdo al rango de probabilidad descripto en la tabla 1 , que van de un rango de 1 al 5 , y a las categorías de severidad que se presenta en la tabla 2, que van de un rango de 2 al 5. 
Percepción de riesgo ante eventos de salud urbana en trabajadores de epidemiología en Paraguay

Tabla 1.

Eventos de riesgo para la salud en el contexto urbano

\begin{tabular}{|c|c|}
\hline Evento & Riesgo \\
\hline Ébola & $\begin{array}{l}\text { Los brotes de enfermedad por el virus del Ebola (EVE) } \\
\text { tienen una tasa de letalidad que puede llegar al } 90 \% \text {. } \\
\text { El brote que inició en } 2014 \text { en África Occidental fue } \\
\text { calificado por la OMS como una emergencia de salud } \\
\text { pública internacional. Hasta mayo de } 2015 \text { se habían } \\
\text { registrado casi } 27.000 \text { casos y más de } 11.000 \\
\text { fallecidos (OMS, 2015) }\end{array}$ \\
\hline Dengue & $\begin{array}{l}\text { El dengue es la infección vírica transmitida por } \\
\text { vectores de mayor propagación en el mundo y } \\
\text { representa un problema grave de salud pública. En la } \\
\text { región de las Américas, entre el } 2008 \text { y el } 2012 \text { se } \\
\text { notificaron más de } 1,2 \text { millones de casos anuales, } \\
\text { incluidos más de } 28.000 \text { casos graves y } 1.000 \text { muertes } \\
\text { (OPS/OMS,2014). }\end{array}$ \\
\hline Chikungunya & $\begin{array}{l}\text { La fiebre chikungunya es una enfermedad vírica } \\
\text { transmitida al ser humano por mosquitos infectados. } \\
\text { La enfermedad se reportó por primera vez en la región } \\
\text { de las Américas en diciembre de } 2013 \text {. Desde } \\
\text { entonces, hasta mayo de } 2015 \text {, se habían reportado } \\
\text { casi } 1,5 \text { millones de casos en la región. (OPS/OMS, } \\
2015 \text { ) }\end{array}$ \\
\hline Obesidad & $\begin{array}{l}\text { La obesidad ha alcanzado proporciones epidémicas a } \\
\text { nivel mundial. Cada año mueren, como mínimo, } 2.6 \\
\text { millones de personas por sus complicaciones. El } \\
\text { sedentarismo y estilo de alimentación urbano } \\
\text { contribuye a su masificación. }\end{array}$ \\
\hline VIH & $\begin{array}{l}\text { Alrededor de } 1,8 \text { millones de personas vivían con el } \\
\text { VIH en América Latina y el Caribe en } 2012 \text {. Ese año } \\
\text { hubo aproximadamente } 98.000 \text { nuevas infecciones. La } \\
\text { OPS/OMS, calcula que el } 70 \% \text { de las personas que } \\
\text { viven con VIH en la región conocen que están } \\
\text { infectadas (OPS/OMS, 2014) }\end{array}$ \\
\hline $\begin{array}{l}\text { Infecciones } \\
\text { respiratorias } \\
\text { agudas } \\
\text { graves - } \\
\text { IRAG }\end{array}$ & $\begin{array}{l}\text { Se estiman que se producen entre } 250.000 \text { a } 500.000 \\
\text { muertes por año debido a la influenza. Las tasas de } \\
\text { hospitalización por IRAG asociadas a la influenza y la } \\
\text { mortalidad son más altas en los menores de } 5 \text { años y } \\
\text { más de } 64 \text { años de edad. }\end{array}$ \\
\hline $\begin{array}{l}\text { Asaltos } \\
\text { violentos }\end{array}$ & $\begin{array}{l}\text { En América Latina la violencia es extensa y tiene } \\
\text { inmensos costos. En la región hay } 140.000 \text { homicidios } \\
\text { y } 28 \text { millones de familias son sujetas a hurto o robo } \\
\text { cada año (Londoño y Guerrero, 1999). }\end{array}$ \\
\hline
\end{tabular}


Percepción de riesgo ante eventos de salud urbana en trabajadores de epidemiología en Paraguay

Tabla 2.

Rango de probabilidad de un evento

\begin{tabular}{lll}
\hline Nivel & Descripción & Detalle \\
\hline 5 & Muy frecuente & $\begin{array}{l}\text { El problema estará presente muchas veces, durante } \\
\text { toda la vida }\end{array}$ \\
4 & $\begin{array}{l}\text { Bastante } \\
\text { frecuente }\end{array}$ & $\begin{array}{l}\text { El problema aparecerá muchas veces a lo largo de la } \\
\text { vida } \\
\text { Este problema se puede presentar en forma ocasional } \\
\text { alguna vez en la vida }\end{array}$ \\
3 & Ocasional & Es poco probable que el problema se presente \\
1 & Remota & $\begin{array}{l}\text { Es tan improbable que ni siquiera vale la pena } \\
\text { considerarlo }\end{array}$ \\
\hline
\end{tabular}

En la adaptación de la herramienta de evaluación del riesgo se advirtió que este tipo de escalas de medición de probabilidad del evento está construido principalmente para eventos inusuales, por lo que su aplicación a eventos de carácter habitual requirió ajustes.

La adecuación consistió en diferenciar la probabilidad personal y colectiva de ocurrencia del evento. Si se considera la probabilidad personal, se refiere a la probabilidad que tiene una persona en particular de sufrir el daño evaluado. Si se piensa como una probabilidad colectiva, se piensa en la probabilidad de que el evento adquiera dimensiones para ser considerado un problema de salud pública.

La evaluación del riesgo estaría determinada por ambos factores, tanto por la probabilidad individual de sufrir una amenaza por las consecuencias directas que tiene la enfermedad, como por la probabilidad de que el evento se convierta en un problema colectivo, aun cuando la persona en sí no sea directamente afectada.

El modelo de evaluación de riesgos aproximados contiene una matriz para la clasificación de riesgos, que se construye a partir de las variables previamente descriptas (Tabla 3). La misma se complementa con un índice de clasificación del riesgo (Tabla 4), que se obtiene al multiplicar probabilidad $(P)$ por la severidad $(S)$; siendo $\mathrm{R}=\mathrm{P} \times \mathrm{S}$. A partir de allí se construye una variable del tipo ordinal, donde se clasifican los riesgos en cuatros tipos: muy altos, altos, moderados y bajos (tabla 5).

Revista de Comunicación y Salud, 2017, Vol. 7, pp. 61-79 
Percepción de riesgo ante eventos de salud urbana en trabajadores de epidemiología en Paraguay

Tabla 3.

Categorías de severidad de un evento

\begin{tabular}{lll}
\hline Nivel & Descripción & Detalle \\
\hline 4 & Desastroso & $\begin{array}{l}\text { Puede ocasionar muertes o un colapso en el sistema de } \\
\text { salud }\end{array}$ \\
3 & Crítico & $\begin{array}{l}\text { Puede ocasionar serios problemas en las personas o el } \\
\text { sistema de salud } \\
\text { Puede ocasionar problemas menores en las personas o en } \\
\text { el sistema de salud }\end{array}$ \\
2 & Insignificante & $\begin{array}{l}\text { El daño que puede ocasionar en las personas o en el } \\
\text { sistema de salud es pequeño }\end{array}$ \\
\hline
\end{tabular}

Tabla 4.

Matriz de clasificación del riesgo

\begin{tabular}{lcccc}
\hline \multicolumn{1}{c}{$\begin{array}{c}\text { Probabilidad del } \\
\text { evento }\end{array}$} & \multicolumn{4}{c}{ Severidad } \\
\cline { 2 - 5 } & Desastroso & Crítico & Insignificante & Desatendible \\
\hline Muy frecuente & 16 & 12 & 10 & 5 \\
Probable & 12 & 9 & 8 & 4 \\
Ocasional & 8 & 6 & 4 & 3 \\
Remota & 4 & 3 & 2 & 2 \\
Improbable & & & & 1 \\
\hline
\end{tabular}

Tabla 5.

Índice de evaluación del riesgo

\begin{tabular}{ccl}
\hline Valor & Tipo & Categoría \\
\hline 20 a 12 & 4 & Es un riesgo muy alto, inaceptable para la población \\
8 a 10 & 3 & Es un riesgo alto, indeseable para la población \\
4 a 6 & 2 & $\begin{array}{l}\text { Es un riesgo moderado, aceptable, pero que debe ser } \\
\text { evaluado }\end{array}$ \\
1 a 3 & 1 & Es un riesgo bajo, aceptable, que no necesita ser evaluado \\
\hline
\end{tabular}

Revista de Comunicación y Salud, 2017, Vol. 7, pp. 61-79 
Percepción de riesgo ante eventos de salud urbana en trabajadores de epidemiología en Paraguay

En este estudio se propuso analizar el grado de correlación entre la percepción de riesgo de los siete eventos de salud urbana previamente descriptos (riesgo subjetivo), y el índice de evaluación de riesgo resultante de combinar el valor asignado a la magnitud y probabilidad de los mismos eventos (riesgo objetivo).

La variable sobre la que se contrastó el índice construido fue el grado de percepción de riesgo de los encuestados para cada evento. Para conocerlo se incluyó en el cuestionario una pregunta inicial donde los participantes calificaron espontáneamente el riesgo que a su criterio representa para el país, cada uno de los eventos de salud pública. La pregunta fue construida de la siguiente forma: "En la siguiente tabla, se presentan eventos que de alguna manera están asociados con la salud pública en un contexto urbano. Por favor, califique los siguientes problemas según mejor definan el riesgo que representan para el país". El participante debía calificar como: muy alto, alto, moderado o bajo.

Posteriormente en el cuestionario, se le solicitó al participante evaluar el riesgo de los mismos problemas, considerando su magnitud y frecuencia de ocurrencia. Se le pidió asignar valores de probabilidad de ocurrencia a nivel personal, probabilidad de ocurrencia a nivel colectivo, magnitud del problema a nivel personal y magnitud del evento a nivel colectivo, de acuerdo a la escala presentada previamente.

Se incluyeron preguntas para medir los componentes significativos en la percepción de riesgo (Covello y Merkhoher, 1993) específicamente redactadas para medir la dimensión en cada uno de los eventos evaluados.

Se incluyeron además preguntas de identificación de los participantes, sobre su práctica profesional y sobre el uso de medios para informarse sobre temas en general y sobre temas específicos de salud.

Antes de su distribución a los participantes, el cuestionario fue previamente revisado por expertos en el área de salud pública, epidemiología, estadística y comunicación de riesgo, que sugirieron ajustes que fueron incluidos en la versión final.

\subsection{Análisis de los datos}

Se realizó el análisis descriptivo de la muestra para las variables demográficas y de acceso de información. Se utilizó el formulario de Google para la recolección de datos y el software SPSS, en su versión 22 para el análisis.

Con los valores asignados a la probabilidad y magnitud de cada evento a nivel personal y colectivo, los investigadores construyeron los índices de evaluación de riesgo utilizando el modelo propuesto, que se compararon con los valores de percepción de riesgo asignados por los participantes a los mismos eventos.

Se comprobó mediante el test de Shapiro-Wilk que las variables de percepción y evaluación de riesgo, para cada una de las enfermedades, no seguían una distribución normal.

Se calculó, por tanto, el coeficiente de correlación de Spearman para variables no paramétricas, con el objetivo de identificar si las variables de evaluación de riesgo

Revista de Comunicación y Salud, 2017, Vol. 7, pp. 61-79 
Percepción de riesgo ante eventos de salud urbana en trabajadores de epidemiología en Paraguay

personal y colectivo estaban correlacionadas. Se utilizó, seguidamente, la prueba no paramétrica de Kruskal-Wallis para comparar si la evaluación de riesgo (tanto personal como colectiva) era distinta a la percepción de riesgo para cada uno de los eventos.

En un segundo momento, se aplicó la prueba $U$ de Mann-Whitney para verificar si alguno de los factores asociados en la literatura con la percepción de riesgo estaban relacionados con las variables de percepción y evaluación de riesgo. Algunos de estos factores, de acuerdo a la literatura, estarían asociados a un aumento y otros a una reducción en la percepción de riesgo, según fue construida la pregunta en el cuestionario. Se tuvo en cuenta la dirección de la pregunta en el análisis.

Se verificó si existía alguna relación entre la percepción de riesgo y la edad, a través del coeficiente de relación de Spearman, y si había diferencia en la percepción o en la evaluación de riesgo para los epidemiólogos en comparación con otros grupos profesionales, utilizando la prueba $U$ de Mann-Whitney.

\section{RESULTADOS}

Se analizaron un total de 32 encuestas, lo que representó una tasa de respuesta del $19,5 \%$ y una representatividad de alrededor del $10 \%$ de los funcionarios de la red de vigilancia de la salud en Paraguay. El análisis de las variables demográficas, se observan en la tabla 6.

Tabla 6.

Descripción de las participantes del estudio sobre percepción de riesgo entre trabajadores de la Red de Vigilancia de la Salud ( $n=32)$

\begin{tabular}{|c|c|c|}
\hline \multicolumn{3}{|l|}{ Edad (en años) } \\
\hline Media & 37,2 & \\
\hline Mediana & 35 & \\
\hline Rango & $\begin{array}{c}24- \\
58\end{array}$ & \\
\hline Sexo & $\mathrm{n}$ & $\%$ \\
\hline Femenino & 26 & 81,3 \\
\hline Masculino & 6 & 18,8 \\
\hline \multicolumn{3}{|l|}{ Formación } \\
\hline $\begin{array}{l}\text { Técnico/Universitario } \\
\text { incompleto }\end{array}$ & 3 & 9,4 \\
\hline Universitario completo & 11 & 34,4 \\
\hline $\begin{array}{l}\text { Postgrado (Diplomado, } \\
\text { maestría, doctorado) }\end{array}$ & 17 & 53,1 \\
\hline \multicolumn{3}{|l|}{ Lugar de residencia } \\
\hline Asunción & 19 & 59,4 \\
\hline Gran Asunción* & 9 & 28,1 \\
\hline Otro & 4 & 12,5 \\
\hline
\end{tabular}

Revista de Comunicación y Salud, 2017, Vol. 7, pp. 61-79 
Percepción de riesgo ante eventos de salud urbana en trabajadores de epidemiología en Paraguay

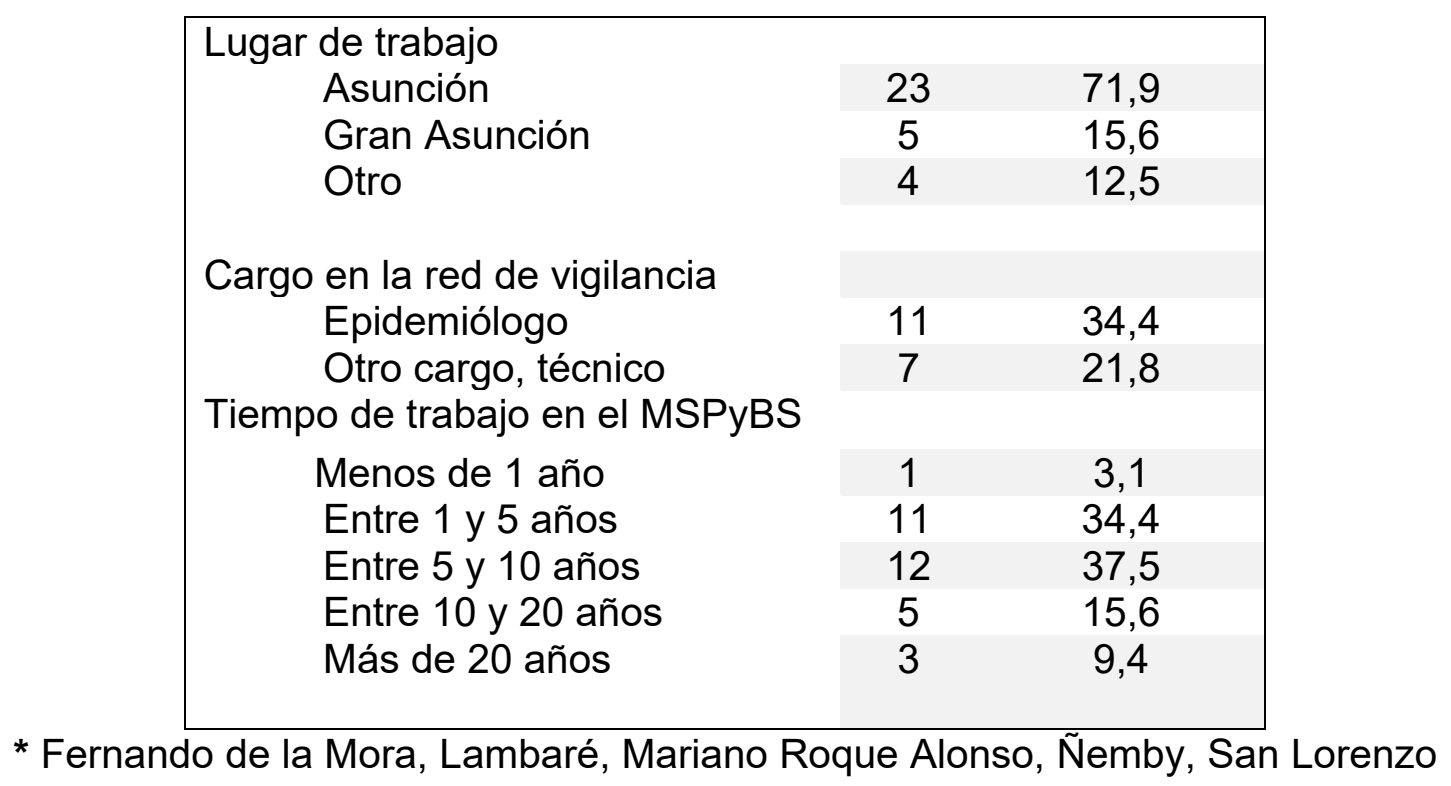

\subsection{Preferencias de medios de información}

Con respecto al acceso a información, se identificó preferencia por los medios digitales (redes sociales y prensa online), que fueron citados entre las primeras tres opciones de información en el $78,1 \%$ y $75 \%$ de los participantes. Específicamente como primera opción, los prefirió el $53,2 \%$ y $40,6 \%$, respectivamente. Los detalles se ven en la tabla 7. Los boletines electrónicos y el correo electrónico constituyeron los medios citados con mayor frecuencia como primera opción $(43,8 \%)$, después de las redes sociales $(53,2 \%)$.

Como medios de información sobre temas específicos de salud, los participantes citaron libremente su preferencia por: sitios web de instituciones de salud (especialmente la web del Ministerio de Salud) y revistas científicas, boletines epidemiológicos, redes sociales, prensa escrita, y los colegas de trabajo.

Tabla 7.

Uso de medios de información entre trabajadores de la red de vigilancia de la salud. $n=32$

\begin{tabular}{|c|c|c|c|c|c|c|c|c|}
\hline & \multicolumn{2}{|c|}{ Total } & \multicolumn{2}{|c|}{$\begin{array}{l}\text { Primera } \\
\text { opción }\end{array}$} & \multicolumn{2}{|c|}{$\begin{array}{c}\text { Segunda } \\
\text { opción }\end{array}$} & \multicolumn{2}{|c|}{$\begin{array}{l}\text { Tercera } \\
\text { opción }\end{array}$} \\
\hline & $n$ & $\%$ & & $\%$ & & $\%$ & & $\%$ \\
\hline $\begin{array}{l}\text { Redes sociales (Facebook, } \\
\text { whatsapp, otras) }\end{array}$ & 25 & 78,1 & 17 & 53,1 & & 9,4 & 5 & 15,6 \\
\hline $\begin{array}{l}\text { Internet (sitios web, blogs, prensa } \\
\text { online) }\end{array}$ & 24 & 75,0 & 13 & 40,6 & 9 & 28,1 & 2 & 6,3 \\
\hline $\begin{array}{l}\text { Televisión (canales por cable o } \\
\text { aire) }\end{array}$ & 21 & 65,6 & 9 & 28,1 & 6 & 18,8 & 6 & 18,8 \\
\hline $\begin{array}{l}\text { Boletines electrónicos, correo } \\
\text { electrónico }\end{array}$ & 21 & 65,6 & 14 & 43,8 & 4 & 12,5 & 3 & 9,4 \\
\hline
\end{tabular}


Percepción de riesgo ante eventos de salud urbana en trabajadores de epidemiología en Paraguay

\begin{tabular}{|c|c|c|c|c|c|c|c|c|}
\hline Hablando con otras personas & 17 & 53,1 & 8 & 25,0 & 5 & 15,6 & 4 & 12,5 \\
\hline Radio & 15 & 46,8 & 8 & 25,0 & 4 & 12,5 & 3 & 9,4 \\
\hline $\begin{array}{l}\text { Prensa impresa (diarios, revistas, } \\
\text { etc.) }\end{array}$ & 13 & 40,6 & 7 & 21,9 & 2 & 6,3 & 4 & 12,5 \\
\hline Televisión (on line) & 12 & 37,5 & 6 & 18,8 & 2 & 6,3 & 4 & 12,5 \\
\hline
\end{tabular}

\subsection{Relación entre percepción y evaluación de riesgo}

Existía una correlación entre percepción y evaluación de riesgo a nivel colectivo, para diferentes eventos de salud urbana (Ébola coeficiente de correlación de Spearman: $0,37$; Chikungunya 0,43 ; Obesidad 0,49 y VIH 0,54$)$ pero solo con el riesgo de Asaltos, en la evaluación de riesgo personal $(0,53)$. Con respecto a la comparación entre percepción y evaluación de riesgo, se identificó que existían diferencias significativas en la evaluación de riesgo colectiva, en comparación con la percepción de riesgo expresada por los participantes (Tabla 8).

Tabla 8.

Percepción y evaluación de riesgo ante eventos de salud urbana, en trabajadores de la red de vigilancia de la salud. $n=32$

\begin{tabular}{lc|clcl}
\hline Evento & $\begin{array}{c}\text { Mediana } \\
\text { Percepción } \\
\text { de riesgo }\end{array}$ & $\begin{array}{c}\text { Mediana de } \\
\text { evaluación } \\
\text { de riesgo } \\
\text { personal }\end{array}$ & Sig & $\begin{array}{c}\text { Mediana de } \\
\text { evaluación } \\
\text { de riesgo } \\
\text { colectiva }\end{array}$ & Sig. \\
\hline Ébola & 1 & 3 & 0,20 & 3 & 0,22 \\
Dengue & 3 & 4 & 0,27 & 4 & $0,01^{*}$ \\
Chikungunya & 3 & 3 & 0,26 & 4 & $0,04^{*}$ \\
Obesidad & 3 & 3 & 0,11 & 4 & $0,02^{*}$ \\
VIH & 3 & 3 & 0,23 & 4 & $0,004^{*}$ \\
IRAG & 3 & 3 & 0,23 & 4 & 0,05 \\
Asaltos & 3 & 4 & $0,002^{*}$ & 3 & 0,27 \\
\hline
\end{tabular}

En cinco de los siete eventos evaluados la percepción de riesgo fue significativamente más baja que la evaluación del riesgo a nivel colectivo (KruskalWallis, $p<0,05$ ) (dengue, chikungunya, obesidad, $\mathrm{VIH}$ e infecciones respiratorias agudas), y en uno de los eventos (asaltos), la percepción fue más baja que la evaluación de riesgo personal. Ver detalles en Tabla 9. 
Percepción de riesgo ante eventos de salud urbana en trabajadores de epidemiología en Paraguay

Tabla 9.

Correlación entre percepción y evaluación de riesgo a nivel personal y colectivo, para diferentes eventos de salud urbana $(n=32)$.

\begin{tabular}{|c|c|c|c|c|}
\hline \multirow{2}{*}{$\begin{array}{c}\text { Percepción de } \\
\text { riesgo }\end{array}$} & \multicolumn{2}{|c|}{$\begin{array}{l}\text { Evaluación de riesgo } \\
\text { personal }\end{array}$} & \multicolumn{2}{|c|}{ Evaluación de riesgo colectivo } \\
\hline & $\begin{array}{l}\text { personal } \\
\text { Coeficiente } \\
\text { de correlación }\end{array}$ & Sig. & $\begin{array}{l}\text { Coeficiente de } \\
\text { correlación }\end{array}$ & Sig. \\
\hline Ébola & & & $0,37^{*}$ & 0,046 \\
\hline Dengue & & & & \\
\hline Chikungunya & & & $0,43^{*}$ & 0,014 \\
\hline Obesidad & & & $0,49^{* *}$ & 0,005 \\
\hline VIH & & & $0,54^{* *}$ & 0,002 \\
\hline IRAG & & & & \\
\hline Asaltos & $0,53^{* *}$ & 0,004 & & \\
\hline
\end{tabular}

La comparación entre evaluación y percepción de riesgo podía tomar tres tipos distintos para cada evento: a. Percepción de riesgo más baja; b. Percepción de riesgo igual; c. Percepción de riesgo más alta; y para cada nivel de análisis (personal y colectivo). La tabla 10 muestra la comparación entre percepción y riesgo evaluado, para cada evento.

Tabla 10.

Comparación entre percepción y riesgo evaluado, para cada evento en análisis, por nivel de análisis, para las diferencias significativas.

\begin{tabular}{lcc}
\hline & $\begin{array}{c}\text { Percepción de riesgo vs. } \\
\text { Riesgo personal evaluado }\end{array}$ & $\begin{array}{c}\text { Percepción de riesgo vs. } \\
\text { Riesgo Colectivo } \\
\text { evaluado }\end{array}$ \\
\hline Ébola & - & - \\
Dengue & - & Más baja \\
Chikungunya & - & Más baja \\
Obesidad & - & Más baja \\
VIH & - & Más baja \\
IRAG & - & - \\
Asalto violento & Más baja & - \\
\hline
\end{tabular}

En cinco de los siete eventos evaluados, la percepción de riesgo expresada fue significativamente más baja que la evaluación del riesgo a nivel colectivo.

No se identificó diferencia significativa entre la percepción y evaluación de riesgo de los participantes, según su edad ni según el cargo desempeñado. (Tabla 11) 
Percepción de riesgo ante eventos de salud urbana en trabajadores de epidemiología en Paraguay

Tabla 11

Percepción del riesgo de los eventos de salud según cargo que desempeñan los entrevistados

\begin{tabular}{lc|c|c}
\hline & \multicolumn{3}{c}{ Mediana Percepción de riesgo } \\
\cline { 2 - 4 } Evento & $\begin{array}{c}\text { Epidemiólogos } \\
\mathrm{n}=10\end{array}$ & $\begin{array}{c}\text { Otro Cargo } \\
\mathrm{n}=22\end{array}$ & Sig. \\
\hline Ébola & 1 & 1,5 & 0,48 \\
Dengue & 3,5 & 3 & 0,56 \\
Chikungunya & 3 & 3 & 0,89 \\
Obesidad & 3 & 3 & 0,59 \\
VIH & 3,5 & 3 & 0,18 \\
IRAG & 3 & 3 & 0,66 \\
Asaltos & 3 & 3 & 0,95 \\
\hline
\end{tabular}

\subsection{Factores relacionados con la percepción de riesgo}

En un segundo momento, se buscó si existían factores que estuvieran relacionados con la percepción y evaluación de riesgo del evento.

En las tablas 12 y 13 se muestra el porcentaje de respuestas positivas a la presencia de factores que según la literatura están asociados con una mayor o menor percepción de riesgo.

En este trabajo se identificó significancia estadística en algunos de los factores, para algunas de las enfermedades. El factor donde se identificó asociación en más cantidad de eventos fue la "percepción de vulnerabilidad", seguido del "temor" y la "posibilidad de catástrofe".

Tabla 12

Presencia de factores que según la literatura están asociados a una reducción en la percepción de riesgo.

\begin{tabular}{lccccc}
\hline & $\begin{array}{c}\text { Conocimiento } \\
\text { sobre el } \\
\text { evento }\end{array}$ & $\begin{array}{c}\text { Comprensión } \\
\text { del riesgo }\end{array}$ & $\begin{array}{c}\text { Control } \\
\text { sobre el } \\
\text { riesgo }\end{array}$ & $\begin{array}{c}\text { Reversibilidad } \\
\text { del daño }\end{array}$ & $\begin{array}{c}\text { Confianza } \\
\text { en las } \\
\text { autoridades }\end{array}$ \\
\hline Ébola & $\%$ & $\%$ & $\%$ & $\%$ & $\%$ \\
Dengue & 81,3 & 100 & 84,4 & 31,3 & 68,8 \\
Chikungunya & 100 & 100 & 93,8 & $93,8 * \mathrm{~b}$ & $62,5 * \mathrm{c}$ \\
Obesidad & 100 & 100 & 93,8 & 90,6 & 62,5 \\
VIH & 87,5 & 100 & 100 & 96,9 & 43,8 \\
IRAG & 96,9 & 100 & 100 & 40,6 & 71,9 \\
Asaltos & $93,8 * a$ & 96,9 & 78,1 & 93,8 & 65,6 \\
\hline a & 96,9 & 90,6 & 46,9 & 78,1 & 15,6 \\
\hline
\end{tabular}

${ }^{\mathrm{a}}$ Sig. $=0,027$ para Evaluación de riesgo colectivo

${ }^{\mathrm{b}}$ Sig. $=0,003$ para Evaluación de riesgo colectivo

${ }^{c}$ Sig. $=0,041$ para Evaluación de riesgo individual 
Percepción de riesgo ante eventos de salud urbana en trabajadores de epidemiología en Paraguay

Tabla 13

Presencia de factores que según la literatura están asociados a un aumento en la percepción de riesgo.

\begin{tabular}{|c|c|c|c|c|c|c|}
\hline & $\begin{array}{l}\text { Incertidumbre } \\
\text { sobre la } \\
\text { probabilidad } \\
\text { de ser } \\
\text { afectado } \\
\end{array}$ & $\begin{array}{l}\text { Existencia } \\
\text { de grupos } \\
\text { más } \\
\text { afectados }\end{array}$ & $\begin{array}{l}\text { Temor } \\
\text { hacia } \\
\text { el } \\
\text { evento }\end{array}$ & $\begin{array}{c}\text { Percepción } \\
\text { de } \\
\text { vulnerabilidad }\end{array}$ & $\begin{array}{l}\text { Experiencia } \\
\text { personal }\end{array}$ & $\begin{array}{c}\text { Posibilidad } \\
\text { de } \\
\text { Catástrofe }\end{array}$ \\
\hline & $\%$ & $\%$ & $\%$ & $\%$ & $\%$ & $\%$ \\
\hline Ébola & 18,8 & 75,0 & 59,4 & 96,9 & 0 & 31,3 \\
\hline Dengue & 71,9 & 68,8 & $\underset{* b}{71,9}$ & 93,8 & 100 & $93,8 * k$ \\
\hline Chikungunya & 68,8 & 68,8 & 68,8 & 90,6 & 71,9 & 90,6 \\
\hline Obesidad & 65,6 & 78,1 *a & $50 * c$ & $78,1^{* d e}$ & 96,9 & 81,3 \\
\hline VIH & 40,6 & 84,4 & 71,9 & $43,8 * f g$ & 68,8 & $46,9 * 1$ \\
\hline IRAG & 65,6 & 78,1 & 75 & $75 * h \mathrm{i}$ & 78,1 & 50 \\
\hline Asaltos & 68,8 & 71,9 & 96,9 & $87,5^{* j}$ & 71,9 & 65,6 \\
\hline $\begin{array}{l}{ }^{a} \text { Sig. }=0,042 \text { para } \\
\text { personal } \\
\text { c Sig. }=0,013 \\
\text { personal } \\
\text { dig. }=0,018 \\
\text { personal } \\
\text { Sig. }=0,030 \text { para } \\
\text { h Sig. }=0,018 \\
\text { personal } \\
\text { Sig. }=0,016 \text { pare }\end{array}$ & $\begin{array}{l}\text { Evaluación de ries } \\
\text { para Evaluación } \\
\text { para Evaluación } \\
\text { Evaluación de ries } \\
\text { para Evaluación } \\
\text { a Percepción de rie }\end{array}$ & $\begin{array}{l}\text { de riesgo } \\
\text { de riesgo } \\
\text { sgo personal } \\
\text { de riesgo } \\
\text { esgo }\end{array}$ & \multicolumn{4}{|c|}{$\begin{array}{l}\text { e Sig. }=0,025 \text { para Percepción de riesgo } \\
\text { 'Sig. }=0,042 \text { para Percepción de riesgo } \\
{ }^{g} \text { Sig. }=0,009 \text { para Evaluación de riesgo colectivo } \\
\text { 'Sig. }=0,05 \text { para Evaluación de riesgo colectivo } \\
\text { j Sig. }=0,019 \text { para Evaluación de riesgo colectivo } \\
{ }^{k} \text { Sig. }=0,000 \text { para Evaluación de riesgo colectivo }\end{array}$} \\
\hline
\end{tabular}

\section{DISCUSIÓN}

En el presente estudio se identificó que existe correlación entre la percepción y el índice de evaluación de riesgo que se construyó a partir de las respuestas de los participantes del estudio sobre probabilidad y gravedad del evento de salud desde el punto de vista colectivo.

Conocer la percepción de riesgo de profesionales del área de epidemiología no es un tema menor, ya que el juicio de este grupo profesional está vinculado a la respuesta al riesgo. Según uno de los marcos de evaluación de políticas públicas de desarrollo más utilizados (Presión-Estado-Respuesta) las actividades humanas ejercen presiones sobre el ambiente y generan cambios en el estado de los ecosistemas, a los que las sociedades responden a través de políticas ambientales, económicas y programas para reducir, prevenir o mitigar estos riesgos. En un sentido amplio, estos pasos forman pasos de un ciclo, que incluye la percepción del problema, la formulación, el monitoreo y la evaluación de políticas (OECD, 1993). 
Percepción de riesgo ante eventos de salud urbana en trabajadores de epidemiología en Paraguay

La percepción pública del riesgo puede ser exagerada y forzar medidas injustificadas, y esto puede generar gastos innecesarios (Philp, 2013). Su entendimiento constituye por tanto una prioridad. En este estudio, sin embargo, la percepción de riesgo para el personal del área de epidemiología resultó más baja con respecto al índice de evaluación de riesgo, en la mayoría de los eventos evaluados. En contrapartida, no se encontraron diferencias entre la percepción de riesgo y el índice de evaluación de riesgo en el nivel personal en la mayoría de los eventos.

La percepción de riesgo más baja en este estudio podría estar relacionada con el tipo de población a la que estuvo dirigida la encuesta. Se trata de profesionales del área de la salud dedicados a la vigilancia y control de eventos de riesgo, donde la exposición rutinaria de información sobre estos temas, podría estar asociada a una reducción en la perspectiva del riesgo. No se identificaron diferencias significativas en las respuestas de epidemiólogos especialistas en el área, aunque por algunos indicios en la orientación de la respuesta podría ser interesante aumentar el número de muestras para ver si las diferencias se vuelven significativas. Se debe tener en cuenta que la percepción de riesgo es un factor importante para la respuesta al problema, dado que puede determinar que se apliquen o no medidas de protección (Rasmussen, 2012). Por tanto, para este grupo particular, podría ser interesante profundizar en la identificación de factores que se relacionen con la baja percepción observada.

Sería interesante, además, contrastar las respuestas de este grupo de población con el de otras poblaciones clave, tales como los funcionarios de gobiernos locales, periodistas, docentes, entre otros. También sería interesante contrastar con grupos de diferente nivel educativo, considerando que el nivel educativo de esta población es alto $(34,4 \%$ tiene universitario completo y $53,1 \%$ tiene estudios de postgrados).

\section{CONCLUSIÓN}

Los eventos seleccionados constituyen eventos relevantes desde la perspectiva de la salud urbana para los participantes, considerando que fueron mayormente calificados como de alto y muy alto riesgo. No hay que olvidar que el entorno urbano tiene una influencia importante en la organización y planificación de la vida social, determinan su estilo de vivienda y sus redes sociales (Nottridge, 2013) Una característica particular del grupo estudiado, es que el $31,2 \%$ de los encuestados vivía y trabajaba en ciudades distintas (la modalidad más frecuente es vivir en Gran Asunción y trabajar en Asunción, aunque también se observó el caso inverso). Esto implica la necesidad de dedicar más tiempo al desplazamiento, y también podría estar relacionado a estar más expuesto a ciertos eventos y menos expuesto a otros.

Otra característica interesante resultó la alta frecuencia de uso de redes sociales y medios online, como fuentes de información sobre temas generales y de salud, lo que lleva a analizar el comportamiento en su contexto socio-físico (Stokols y Clitheroe, 2010) y cómo el concepto de comunidad ha cambiado, no limitándose a la proximidad geográfica que se observa en ambientes rurales. En los ambientes urbanos, cada vez más, la comunidad se crea por las redes sociales que construye la 
Percepción de riesgo ante eventos de salud urbana en trabajadores de epidemiología en Paraguay

persona en su día a día, y constituyen esto un medio de información importante. En este estudio se confirma esta tendencia.

La "nueva comunidad" se define como un conjunto de esas personas, lugares y tecnologías que permiten las interacciones sociofísicas que definen la vida cotidiana. Esta definición asume que los lazos psicológicos de las personas con el medio ambiente local, basadas en el lugar son una fuente importante de su identidad y el bienestar, pero también reconoce que el número y alcance de cercanías psicológicamente significativas de los individuos (como los basados en el hogar, en la escuela, en el trabajo o en entornos comunitarios públicos). Los límites se amplían, ya que ahora puede estar estrechamente vinculados entre sí a través de las comunicaciones digitales en Internet y móviles (Wellman y Haythornthwaite, 2002). Esto obliga a repensar las estrategias de abordaje para afrontar problemas de salud urbana, especialmente las estrategias de comunicación.

Con respecto a los factores citados en la bibliografía de comunicación de riesgo para justificar una mayor percepción de riesgo, muchos de ellos no resultaron significativos para varios de los eventos en este estudio.

En la encuesta, se dio la opción a los participantes a mencionar otros factores que a su criterio aumentan la percepción de riesgo. Entre ellos, se citaron: "grupos de edades afectadas" (por ejemplo, niños), "presencia de factores ambientales que favorezcan la dispersión del evento", "modo de transmisión del evento", "acceso a medios de comunicación", "presencia de factores de riesgo (por ejemplo, diabetes, hipertensión)", "limitaciones en la capacidad para responder al evento (por ejemplo, poca infraestructura en centros asistenciales y personal poco capacitado". Sería favorable complementar el presente estudio, con un estudio del tipo cualitativo, para profundizar sobre estos factores y medir su real impacto sobre los niveles de percepción y evaluación del riesgo a eventos de salud.

La salud de una comunidad está íntimamente ligada con su desarrollo sostenible. Constituye tanto su base, como su producto. Si partimos de la base que el desarrollo sostenible puede ser entendido como una "mejora cualitativa de una base económica física que se mantiene en un estado de equilibrio, mediante un rendimiento material y energético que está dentro de las capacidades regenerativas y asimilación del ecosistema" (Daly, 2010), las estrategias efectivas de comunicación de riesgo en salud son fundamentales para desarrollar respuestas sociales más efectivas y coordinadas. Comprender la forma en que los actores perciben y evalúan el riesgo, contribuye a este objetivo.

\section{BIBLIOGRAFÍA}

Adam, B. (2000). The risk society and beyond: critical issues for social theory. London: SAGE

Berzins, K, Holly Greb, Malea Hoepf Young y Karen Hardee (2014). Urbanization, Population, and Health Myths: Addressing Common Misconceptions with Strategic Health Communication. In Charles C. Okigbo (ed.). Strategic Urban Health Communication. New York: Springer 
Percepción de riesgo ante eventos de salud urbana en trabajadores de epidemiología en Paraguay

Brennan B. y Gutierrez V (2011). Guía para elaborar la estrategia de comunicación de riesgos. Washington, DC: OPS/OMS

Covello, V. y Merkhoher, M. (1993). Risk Assessment Methods: Approaches for Assessing Health and Environmental Risks. New York: Springer

Daly, H. (2010). Sustainable Growth: An Impossibility Theorem. Gaian Economics living well within planetary limits. United Kingdom: Gaia Education. Recuperado de http://www.gci.org.uk/Documents/Gaian Economics.pdf\#page=29

Dye, C. (2008) Health and urban living. Science, 319(5864), 766-769

Flaus G. (2012). A modelling framework for model based risk analysis. Advances in Safety, Reliability and Risk Management . Bérenguer, Grall \& Guedes Soares (eds). London: Taylor \& Francis Group,

Frumkin, H., Frank, LD, y Jackson, R. (2004). Urban sprawl and public health: Designing, planning, and building for healthy communities. Washington, DC: Island Press.

Gordis (2014). Epidemiology. Philadelphia: Saunders.

Harpham, Trudy (2009) Urban health in developing countries: What do we know and where do we go? Health and Place. 15(1), 107-116.

IPCC (2013). Intergovernmental Panel on Climate Chang. Fifth Assessment Report Glossary. Octubre 2013.

Kudlick, C. Jean. (1988). Disease, public health and urban social relations: perceptions of cholera and the Paris environment, 1830-1850. University of California, Berkeley.

Laugesen, J, Det Norske Veritas, Høvik, Norway, Bodil Aamnes Mostue. (2010). The use of risk and vulnerability analysis in climate change adaptation

Lee, A. C. K.; Maheswaran, R. (2011). The health benefits of urban green spaces: a review of the evidence. Journal of public health 33(2), 212-222

Londoño J y Guerrero R, (1999). Violencia en América Latina. Epidemiología y costos. Washington: Banco Interamericano de Desarrollo

Montgomery Mark R. (2009). Urban Poverty and Health in Developing Countries. Population Reference Bureau.

Mythen, G., and Walklate, S. (2006). Beyond the risk society : critical reflections on risk and human security. Maidenhead, England: Open University Press.

Nottridge, Harold E (2013). The Sociology of Urban Living. Routledge.

OMS (2015). Ebola Situation report. 20 May 2015. Recuperado de http://apps.who.int/iris/bitstream/10665/171812/1/roadmapsitrep 20May15 eng. pdf?ua $=1 \& u a=1 \& u a=1$

OMS, 2005. Normas de comunicación de brotes epidémicos de la OMS. Recuperado de

http://www.who.int/csr/resources/publications/WHO_CDS_2005_28spweb.pdf

OPS/OMS (2014). Últimos adelantos técnicos en la prevención $\bar{y}$ el control del dengue en la Región de las Américas. Informe de reunión, 28 y 29 de mayo de 2014. Washington. 
Percepción de riesgo ante eventos de salud urbana en trabajadores de epidemiología en Paraguay

OPS/OMS (2014). Las nuevas metas 2020 para controlar la epidemia de VIH/sida en América Latina y el Caribe. Recuperado de http://www.paho.org/hq/index.php?option=com content\&view=article\&id=9655\&l temid=40019\&lang $=$ es

OPS/OMS (2015). Number of Reported Cases of Chikungunya Fever in the Americas, by Country or Territory 2015 (to week noted) Epidemiological Week / $E W$.

Recuperado

de

http://www.paho.org/hq/index.php?option=com_docman\&task=doc_view\&ltemid $=270 \&$ gid $=42803 \&$ lang $=e n$.

OPS/OMS (2015). Number of Reported Cases of Chikungunya Fever in the Americas, by Country or Territory 2013-2014 Cumulative cases.

Organización de las Naciones Unidas (2014). World Urbanization Prospects. División de Población del Departamento de Asuntos Económicos y Sociales.

Organization for Economic Cooperation and Development (OECD). (1993). Core Set of indicator for Environmental Performance Reviews: A Synthesis Report by the Group on the State of the Environment. Environment Monographs. OCDE/GD (93)179. Paris.

Ostrom, Lee T.; Wilhelmsen, Cheryl A. (2012). Risk Assessment : Tools, Techniques, and Their Applications. Recuperado de http://www.eblib.com

Paul Slovic (2000). The perception of risk London. Sterling, VA : Earthscan Publications

Philp, Richard B. (2013). Ecosystems and Human Health : Toxicology and Environmental Hazards. Bora Raton, FL.:Taylor \& Francis.

Proske D. (2008) Catalogue of Risks Natural, Technical, Social and Health Risks. Springer-Verlag Berlin Heidelberg

Rasmussen, H. B. (2012). The impact of human and organisational factors on risk perception on Danish production platforms, in C. Bérenguer y C. Guedes Soares (eds). Advances in Safety, Reliability and Risk Managemen. Taylor \& Francis

Reis, Renato B.; Ribeiro, Guilherme S.; Felzemburgh, Ridalva et al. (2008). Impact of Environment and Social Gradient on Leptospira Infection in Urban Slums. Plos neglected tropical diseases 2(4), e228

Renn, O (1992) Concepts of risk: A classification. In: S. Krimsky \& D. Golding D (Hrsg), Social theories of risk (pp. 53-79). London: Praeger

Sandman, P. (2003). The Four Kinds of Risk Communication. Recuperado de http://www.psandman.com/col/4kind-1.htm.

Nancy M. Wells, Gary W. Evans, and Kristin Aldred Cheek

Wells, Nancy M., Evans, Gary W., Cheekm Kristin A. (2010). Environmental Psychology. in Frumkin, H. (ed.). Environmental Health: From Global to Local.

Wellman B and Haythornthwaite, C.A. (ed) (2002). The Internet in everyday life. Malden Mass

World Health Organization (2010). Hidden Cities: Unmasking and Overcoming Health Inequities in Urban Settings. 